\title{
WHITEHEAD GROUPS OF CERTAIN SEMIDIRECT PRODUCTS OF FREE GROUPS
}

\author{
KOO-GUAN CHOO
}

\begin{abstract}
Let $D=F_{1} \times F_{2} \times \cdots \times F_{n}$ be a direct product of $n$ free groups $F_{1}, F_{2}, \cdots, F_{n}, \alpha$ an automorphism of $D$ which leaves all but one of the noncyclic factors in $D$ pointwise fixed, $T$ an infinite cyclic group and $F$ another free group. Let $D \times{ }_{\alpha} T$ be the semidirect product of $D$ and $T$ with respect to $\alpha$ and $\left(D \times{ }_{\alpha} T\right) \times_{\alpha \times i \mathrm{~d} T} F$ the semidirect product of $D \times{ }_{\alpha} T$ and $F$ with respect to the automorphism $\alpha \times$ id $T$ of $D \times{ }_{\alpha} T$ induced by $\alpha$. We prove that the Whitehead group of $\left(D \times_{\alpha} T\right) \times_{\alpha \times i d T} F$ and the projective class group of the integral group ring $Z\left(\left(D \times_{\alpha} T\right) \times_{\alpha \times i \mathrm{~d} T} F\right)$ are trivial. These results extend that of [3].
\end{abstract}

Let $G$ be a group. We denote the Whitehead group of $G$ by Wh $G$ and the projective class group of the integral group ring $Z(G)$ of $G$ by $\widetilde{K}_{0} Z(G)$.

We recall the definition of semidirect product of groups and the definition of twisted group ring. For undefined terminologies used in the paper, we refer to [3] and [4].

Let $\alpha$ be an automorphism of $G$ and $F$ a free group generated by $\left\{t_{\lambda}\right\}$. If $w$ is a word in $t_{\lambda}$ defining an element in $F$, we denote by $|w|$ the total exponent sum of the $t_{\lambda}$ appearing in $w$. The semidirect product $G \times{ }_{\alpha} F$ of $G$ and $F$ with respect to $\alpha$ is defined as follows: $G \times{ }_{\alpha} F=G \times F$ as sets and multiplication in $G \times{ }_{\alpha} F$ is given by $(g, w)\left(g^{\prime}, w^{\prime}\right)=\left(g \alpha^{-|w|}\left(g^{\prime}\right), w w^{\prime}\right)$, for any $(g, w),\left(g^{\prime}, w^{\prime}\right)$ in $G \times{ }_{\alpha} F$. In particular, if $F$ is an infinite cyclic group $T=\langle t\rangle$ generated by $t$, we have the semidirect product $G \times{ }_{\alpha} T$ of $G$ and $T$ with respect to $\alpha$.

Let $R$ be an associative ring with identity and $\alpha$ an automorphism of $R$. Let $F$ be a free group (or free semigroup) generated by $\left\{t_{\lambda}\right\}$. The $\alpha$ twisted group ring $R_{\alpha}[F]$ of $F$ over $R$ is defined as follows: additively $R_{\alpha}[F]=R[F]$, the group ring of $F$ over $R$, so that its elements are finite linear combinations of elements in $F$ with coefficients in $R$. Multiplication in $R_{\alpha}[F]$ is given by $(r w)\left(r^{\prime} w^{\prime}\right)=r \alpha^{-|x|}\left(r^{\prime}\right) w w^{\prime}$, for any $r w, r^{\prime} w^{\prime}$ in $R_{\alpha}[F]$. In particular, if $F$ is a free group (resp. free semigroup) generated by $t$, we

Received by the editors May 25, 1973.

AMS (MOS) subject classifications (1970). Primary 13D15, 16A26, 18F25; Secondary 16A06, 16A54.

Key words and phrases. Whitehead group, projective class group, semidirect product of free groups, twisted group ring.

(c) American Mathematical Society 1974 
have the $\alpha$-twisted finite Laurent series ring $R_{\alpha}[T]$ (resp. the $\alpha$-twisted polynomial ring $\left.R_{\alpha}[t]\right)$.

We note that, if $R$ is the integral group $\operatorname{ring} Z(G)$ of $G$ and $\alpha$ an automorphism of $G$, then $\alpha$ induces an automorphism, denoted again by $\alpha$, of $R=Z(G)$ and there is a standard isomorphism between $R_{\alpha}[F]$ and $Z\left(G \times{ }_{\alpha} F\right)$.

Now, let $D=F_{1} \times F_{2} \times \cdots \times F_{n}$ be a direct product of $n$ free groups $F_{1}, F_{2}, \cdots, F_{n}$ and $\alpha$ an automorphism of $D$ which leaves all but one of the noncyclic factors in $D$ pointwise fixed. In [3], we have proved that $\mathrm{Wh}\left(D \times{ }_{\alpha} T\right)=0$ and $\tilde{K}_{0} Z\left(D \times_{\alpha} T\right)=0$, where $T$ is an infinite cyclic group. Let $F$ be another free group. The purpose of this paper is to extend these results to

THEOREM.

$$
\mathrm{Wh}\left(\left(D \times_{\alpha} T\right) \times_{\alpha \times \mathrm{id} T} F\right)=0 \quad \text { and } \quad \widetilde{K}_{0} Z\left(\left(D \times_{\alpha} T\right) \times_{\alpha \times \mathrm{id} T} F\right)=0 \text {, }
$$

where $\alpha \times$ id $T$ is the automorphism of $D \times{ }_{\alpha} T$ induced by $\alpha$.

For simplicity, in the rest of the paper, we will denote by $\alpha^{\mu}$, the automorphism $\alpha^{\mu} \times$ id $T$ of $D \times{ }_{\alpha} T$ induced by the automorphism $\alpha^{\mu}$ on $D$, where $\mu$ is any integer. We need the following result, which is the direct consequence of $[3$, Theorem B] and [4, Theorem 21].

Lemma. Let $D$ and $\alpha$ be as given before the theorem. Then $\tilde{C}\left(Z\left(D \times{ }_{\alpha} T\right), \alpha^{\mu}\right)=0$ for any integer $\mu$.

Proof. Let $T_{1}=\left\langle t_{1}\right\rangle$ be another infinite cyclic group generated by $t_{1}$. Consider the semidirect product $\left(D \times_{\alpha} T\right) \times_{\alpha^{\mu}} T_{1}$. Then by change of generators in $T \times T_{1},\left(D \times{ }_{\alpha} T\right) \times_{\alpha^{\mu}} T_{1}$ can be seen to be isomorphic to $(D \times S) \times_{\alpha \times \text { id } S} T$, where $S=\left\langle t^{-\mu} t_{1}\right\rangle$ is an infinite cyclic group generated by $t^{-\mu} t_{1}$. Then, by [3, Theorem B], $\operatorname{Wh}\left((D \times S) \times_{\alpha \times \operatorname{id} S} T\right)=0$ and so $\mathrm{Wh}\left(\left(D \times{ }_{\alpha} T\right) \times_{\alpha^{\mu}} T_{1}\right)=0$. Hence, it follows from the Farrell-Hsiang decomposition formula for $\operatorname{Wh}\left(\left(D \times_{\alpha} T\right) \times_{\alpha^{\mu}} T_{1}\right)$ (cf. [3, Theorem 1] or [4, Theorem 21]) that $\widetilde{C}\left(Z\left(D \times_{\alpha} T\right), \alpha^{\mu}\right)=0$. This completes the proof.

Next, suppose that $F$ is generated by $\left\{t_{\lambda}\right\}$ and let $R_{\alpha}[F]$ be the $\alpha$-twisted group ring of $F$ over a ring $R$ with automorphism $\alpha$. Notice that, in general, there is no augmentation from $R_{\alpha}[F]$ into $R$. But for certain special rings, such augmentation in fact does exist. Our key step towards the proof of the main theorem is to find such rings so that we can make use of the results in [5].

Now, let $R=Z\left(D \times{ }_{\alpha} T\right)$. Then $R_{\alpha}[F]$ is canonically isomorphic to $Z\left(\left(D \times_{\alpha} T\right) \times_{\alpha} F\right)$. Define a mapping $\varepsilon_{F}: R_{\alpha}[F] \rightarrow R$ by $\varepsilon_{F}\left(r t_{\lambda}\right)=r t$, for all $r$ in $R$ and $t_{\lambda}$ in $F$, where $t$ is the generator of $T$. Then it is clear that 
$\varepsilon_{F}$ is a homomorphism of $R_{\alpha}[F]$ onto $R$ with $\varepsilon_{F}(r)=r$ for all $r$ in $R$, i.e., we can consider $R_{\alpha}[F]$ as an $R$-ring with augmentation $\varepsilon_{F}$ (cf. [5]).

Note that the homomorphism $i_{*}: K_{1} R \rightarrow K_{1} R_{\alpha}[F]$ induced by the inclusion $i: R \rightarrow R_{\alpha}[F]$ is one-to-one, since the homomorphism $\varepsilon_{F^{*}}: K_{1} R_{\alpha}[F] \rightarrow K_{1} R$ induced by $\varepsilon_{F}$, splits $i_{*}$. We will denote the cokernel of the homomorphism $i_{*}$ by $R_{1} R_{\alpha}[F]$. The kernel of $\varepsilon_{F}$ is denoted by $\bar{R}_{\alpha}[F]$, and it is a free $R$-bimodule generated by $w-t^{|w|}$, where $w$ runs over all the words in $t_{\lambda}$, and as bimodules, we have $R_{\alpha}[F]=R \oplus \bar{R}_{\alpha}[F]$. Moreover, if $F^{\prime}$ is another free group, we have $R_{\alpha}\left[F * F^{\prime}\right]=R_{\alpha}[F] * R_{\alpha}\left[F^{\prime}\right]$ (cf. [5]). (Here “*” denotes the free product of groups on the left-hand side and the free product of $R$-rings on the right-hand side.)

Let $M$ be the $R$-bimodule $\bar{R}_{\alpha}[F] \otimes_{R} \bar{R}_{\alpha}\left[F^{\prime}\right]$ and $T_{R}(M)$ the tensor algebra of $M$ over $R$. Let $X$ be the set of noncommuting variables $x$ given by

$$
x=\left(w-t^{|w|}\right) \otimes\left(w^{\prime}-t^{\left|w^{\prime}\right|}\right),
$$

where $w-t^{|w|}$ (resp. $w^{\prime}-t^{\left|w^{\prime}\right|}$ ) runs over the generators of $\bar{R}_{\alpha}[F]$ (resp. $\bar{R}_{\alpha}\left[F^{\prime}\right]$ ) and let $\mathscr{A}=\left\{\alpha^{\mu}\right\}$ ( $\mu$ any integer) be the set of automorphisms $\alpha^{\mu}$ of $R$. Then it is easy to see that $T_{R}(M)$ is nothing but the twisted free associative algebra $R_{\mathscr{A}}\{X\}$ over $X$ (cf. [2]). Since $\widetilde{C}(R, \alpha \mu)=$ $\tilde{C}\left(Z\left(D \times_{\alpha} T\right), \alpha^{\mu}\right)=0$ for any integer $\mu$ (cf. Lemma), it follows from [4, Theorem 13] that $K_{1} R \rightarrow K_{1} R_{\alpha^{\mu}}\left[t^{\prime}\right]$ is an isomorphism for any integer $\mu$, where $R_{\alpha^{\mu}}\left[t^{\prime}\right]$ is the $\alpha^{\mu}$-twisted polynomial ring. Hence, by the twisted case of Gersten's theorem [2, Theorem 2], it follows that $K_{1} R \rightarrow K_{1} T_{R}(M)$ is an isomorphism, i.e. $R_{1} T_{R}(M)=0$. Then, by [5, Theorem 6.1], we have

$$
R_{1} R_{\alpha}\left[F * F^{\prime}\right] \cong R_{1} R_{\alpha}[F] \oplus R_{1} R_{\alpha}\left[F^{\prime}\right]
$$

That is,

$$
\begin{aligned}
R_{1}\left(Z\left(D \times{ }_{\alpha} T\right)\right. & \left.\times_{\alpha}\left(F * F^{\prime}\right)\right) \\
& \cong R_{1}\left(Z\left(D \times_{\alpha} T\right) \times_{\alpha} F\right) \oplus R_{1}\left(Z\left(D \times_{\alpha} T\right) \times_{\alpha} F^{\prime}\right)
\end{aligned}
$$

(We remark that this formula holds for any automorphism $\alpha$ of $D$.)

Finally we give the proof of the theorem. It is similar to that of [3, Lemma 1].

ProOF OF THE THEOREM. First, suppose that $F$ is of infinite rank $m$ and write $F=T_{1} * T_{2} * \cdots * T_{m}$ as a free product of $m$ infinite cyclic groups. Then it follows from (1) that

$$
R_{1} Z\left(\left(D \times_{\alpha} T\right) \times_{\alpha} F\right) \cong \bigoplus_{j=1}^{m} R_{1} Z\left(\left(D \times_{\alpha} T\right) \times_{\alpha} T_{j}\right) .
$$

Using the fact that $\tilde{C}\left(Z\left(D \times_{\alpha} T\right), \alpha^{\mu}\right)=0$ for any integer $\mu$, we deduce 
from [4, Theorem 19] that the sequence

$$
\begin{gathered}
0 \longrightarrow K_{1} Z\left(D \times_{\alpha} T\right) \stackrel{i_{*}}{\underset{\varepsilon_{T_{j}^{*}}}{\longrightarrow}} K_{1} Z\left(\left(D \times_{\alpha} T\right) \times_{\alpha} T_{j}\right) \\
\longrightarrow\left(K_{0} Z\left(D \times{ }_{\alpha} T\right)\right)^{\alpha_{*}} \longrightarrow 0
\end{gathered}
$$

is split short exact, for each $j$. That is

$$
R_{1} Z\left(\left(D \times{ }_{\alpha} T\right) \times_{\alpha} T_{j}\right) \cong\left(K_{0} Z\left(D \times{ }_{\alpha} T\right)\right)^{\alpha^{*}}
$$

for each $j$ and so, it follows from (2) that

$R_{1} Z\left(\left(D \times_{\alpha} T\right) \times_{\alpha} F\right) \cong\left(K_{0} Z\left(D \times_{\alpha} T\right)\right)^{\alpha *} \oplus \cdots \oplus\left(K_{0} Z\left(D \times_{\alpha} T\right)\right)^{\alpha_{*}}$

In other words, the sequence

( $m$ copies).

$$
\begin{aligned}
& 0 \longrightarrow K_{1} Z\left(D \times_{\alpha} T\right) \underset{\varepsilon_{F^{*}}}{\stackrel{i_{*}}{\longleftrightarrow}} K_{1} Z\left(\left(D \times_{\alpha} T\right) \times_{\alpha} F\right) \\
& \longrightarrow\left(K_{0} Z\left(D \times_{\alpha} T\right)\right)^{\alpha_{*}} \oplus \cdots \oplus\left(K_{0} Z\left(D \times_{\alpha} T\right)\right)^{\alpha_{*}} \longrightarrow 0
\end{aligned}
$$

is short exact. Passing to the Whitehead groups, we have (cf. [4])

$$
\begin{aligned}
0 & \rightarrow \mathrm{Wh}\left(D \times{ }_{\alpha} T\right) \rightarrow \mathrm{Wh}\left(\left(D \times_{\alpha} T\right) \times_{\alpha} F\right) \\
& \rightarrow\left(\widetilde{K}_{0} Z\left(D \times_{\alpha} T\right)\right)^{\alpha_{*}} \oplus \cdots \oplus\left(\tilde{K}_{0} Z\left(D \times_{\alpha} T\right)\right)^{\alpha_{*}} \rightarrow 0 .
\end{aligned}
$$

But $\mathrm{Wh}\left(D \times_{\alpha} T\right)=0\left[3\right.$, Theorem B] and $\widetilde{K}_{0} Z\left(D \times_{\alpha} T\right)=0[3$, Theorem A]. Hence $\operatorname{Wh}\left(\left(D \times_{\alpha} T\right) \times_{\alpha} F\right)=0$.

The case when $F$ has infinite rank need not worry us since a matrix over $Z\left(\left(D \times_{\alpha} T\right) \times_{\alpha} F\right)$ involves entries which are sums of words involving only a finite number of free generators of $F$.

Finally, it follows from [4, Theorem 21] and the triviality of Wh $\left(\left(\left(D \times{ }_{\alpha} T\right) \times{ }_{\alpha} F\right) \times T^{\prime}\right)$ (here $T^{\prime}$ denotes another infinite cyclic group) that $\widetilde{K}_{0} Z\left(\left(D \times{ }_{\alpha} T\right) \times_{\alpha} F\right)=0$. This completes the proof.

Since $\left(D \times_{\alpha} T\right) \times_{\alpha \times \mathrm{id} T} F$ and $\left(D \times_{\alpha} F\right) \times_{\alpha \times \mathrm{id} F} T$ are isomorphic, by using $[4$, Theorem 21], we have, in addition to the theorem, that

Corollary. (i) $\tilde{C}\left(Z\left(D \times{ }_{\alpha} F\right), \alpha \times\right.$ id $\left.F\right)=0$.

(ii) $\left(\tilde{K}_{0} Z\left(D \times{ }_{\alpha} F\right)\right)^{(\alpha \times \mathrm{id} F)_{*}}=0$.

(iii) $\mathrm{Wh}\left(D \times{ }_{\alpha} F\right) / I\left((\alpha \times \text { id } F)_{*}\right)=0$.

We still do not know whether or not the factor $T$ in the theorem can be dropped to give triviality of $\operatorname{Wh}\left(D \times_{\alpha} F\right)$. Perhaps it is useful to note that

$$
D \times{ }_{\alpha} F \cong\left(D \times F^{\prime}\right){ }^{*} D\left(D \times{ }_{\alpha} T\right),
$$

where $T=\left\langle t_{\lambda_{0}}\right\rangle$ for some $\lambda_{0}$ and $F^{\prime}=\left\langle t_{\lambda}^{-1} t_{\lambda_{0}}: \lambda \neq \lambda_{0}\right\rangle$. 


\section{REFERENCES}

1. H. Bass, A. Heller and R. G. Swan, The Whitehead group of a polynomial extension, Inst. Hautes Études Sci. Publ. Math. No. 22 (1964), 61-79. MR 30 \#4806.

2. K. G. Choo, Whitehead groups of twisted free associative algebras, Pacific J. Math. (to appear).

3. The projective class group of the fundamental group of a surface is trivial, Proc. Amer. Math. Soc. 40 (1973), 42-46.

4. F. T. Farrell and W. C. Hsiang, A formula for $K_{1} R_{\alpha}[T]$, Applications for Categorical Algebra, Proc. Sympos. Pure Math., vol., 17, Amer. Math. Soc., Providence, R.I., 1970, pp. 192-218. MR 41 \#5457.

5. J. Stallings, Whitehead torsion of free products, Ann. of Math. (2) 82 (1965), 354-363. MR 31 \#3518.

Department of Mathematics, University of British Columbia, Vancouver 8 , British Columbia, Canada 